and the nature of which may be inferred from the fact that I advised the author to withdraw his paper and submit the subject to a renewed investigation, with the aid of improved methods.

With regard to the question, which Mr. Lowne raises, as to my competency to form any opinion at all, on the ground that I had not myself devoted any special attention to the literature of the subject, I may remark that the points which had practically to be decided were (I) whether Mr. Lowne's statements were in themselves probable, and (2) whether they were corroborated by his preparations. Had I not felt myself qualified to form an opinion on these points I should not have accepted the reference.

E. A. SCHÄFER

\section{The Late Prof. Clifford's Papers}

IN the "Mathematical Papers" (pp. 628-37) I was able to print the syllabuses of a series of ten lectures delivered by Prof Clifford to a class of ladies at South Kensington in the spring and summer of $\mathbf{1 8 6 9}$. Whilst turning over a collection of mis cellaneous papers, in a box, Mrs. Clifford and I had the good fortune to light upon a manuscript quite ready for printing, and this ("Mathematical Papers," p. 628) subsequently formed part of the volume on "Seeing and Thinking;" but we could not find any trace of any more manuscript of the above-mentioned series of lectures. Just before the recent Easter holidays Prof. Karl Pearson returned to me a few pages of manuscript bearing on the International Scientific Series volume which $I$ had lent him, and with them he sent me a large note book which had been in the late Prof. Rowe's hands. On opening this book I at once saw that it contained very full notes of other lectures of the course. In fact, Lecture II. ("On Plane Surfaces and Straight Lines") is quite ready for press, as is also, I think, Lecture III. ("On the Rotation of Plane Figures"); Lecture IV. ("Of Similar Figures") is a fragment, and still more fragmentary is Lecture V. " The First Principles of Calculation"). Of Lecture VI. ("The Theorem of Pythagoras") there are two loose sheets of figures : on one sheet is "the Bride's Chair," and the figures on this and the other sheet show that my information was correct, and that the remarks on pp. 633,637 are ad $\mathrm{rcm}$. As Lecture IX. ("On the Shadows of a. Circle") is very fully illustrated in the recent volume edited by Prof. Pearson, we see that we are in possession of a fairly complete presentment of Prof. Clifford's views on the subjects of the course of lectures.

Messrs. Macmillan have stated their willingness to publish the MS. of the second part of "The Elements of Dynamics," and I $\mathrm{h}$ 'pe to be able, after a re-examination of it, to put the work into their hands for printing. When this book is got out, and the above lectures published in some shape yet to be determined, the mathematical world will be in possession of all that we can now look for from the hands of this great master.

University College School, April 25

R. TUCKER

\section{Sir Wm. Thomson and Maxwell's Electro-magnetic Theory of Light}

SHORTLY after writing my former letter I saw a copy of the verbatim report of Sir Wm. Thomson's lectures in Baltimore, and would have written to you to that effect and to apologise to Mr. Forbes for having doubted the accuracy of what I thought was his report, only that I met him in London about that time, and he then desired me not to do so. Sir William Thomson has now himself stated that the passage is correctly quoted, and I can only regret that he has expressed himself in the way he did.

I certainly think that anybody reading the passage would imagine that the velocity of propagation of electro-magnetic disturbances upon Maxwell's electro-magnetic theory of light, which he showed to be the same as the velocity of propagation of light, and to be a true velocity of wave-propagation - any one, I say, would suppose that this was the same thing as that Sir Wm. Thomson calculated in the year 1854

Sir Wm. Thomson certainly says, "That is a very different case," but the rest of this sentence is rather ambiguous as to what the "it" after "putting" refers to, and I am afraid that many people will imagine that, in Sir William Thomson's opinion, Maxwell has made some unjustifiable assumption. I believe, however, that all he thinks is that Maxwell has not made a satisfactorily definite thing of the so-called electro-magnetic theory of light.

In Sir Wm. Thomson's article in Nichol's "Cyclopædia" he puts the matter very clearly indeed. He says:- "The law of this phenomenon [transmission of electric signals] is identical with that which Fourier . . . found as the law of propagation of summer heat and winter cold to different parts of the earth," i.e. it obeys the laws of a diffusion and not of a wave-propagation; and again:- "Now it is obvious from these results [experimental results] that the supposed velocity of transmission of electric signals is not a definite constant like that of light :" and afterwards he says that, when an initial current is started, the potential rises simultaneously at all points, and that the apparent velocity would depend on the delicacy of our instruments. All these obviously distinguish between the propagation of a variable current in a conductor and a true wavepropagation.

He has also clearly pointed out a direction in which to look for a true wave-propagation. It will make his position clearer, and also Maxwell's, to use his analogy between water in an elastic tube and a conductor of electricity. I will suppose the water contained in a tube bored out of a very large lump of india-rubber. He enumerates three electric qualities concerned, and their hydrodynamic analogues:-(I) "Charge" or electrical accumulation in a conductor subjected in any way to the process of electrification. (2) "Electro-magnetic induction" or electromotive force excited in a conductor by variations of electric current. (3) Resistance to conduction through a solid. The hydrodynamic analogues are:-(I) Accumulation of a greater or less quantity of water in any part of the canal or tube. (2) Inertia of the water. (3) Viscosity or fluid friction. $\mathrm{He}$ explains that a true wave-propagation arises from the compressibility of the water, combined with its inertia, and that if the tube be elastic, like india-rubber, there would also arise a wavepropagation. "Accordingly," he says, "a definite velocity of propagation of electric impulses, depending on the inertia and the capacity for charge, is to be looked for, as has been done in a first article, published by Kirchhoff, on the subject."

Now, in all this äiscussion Sir Wm. Thomson omits to mention the only thing that is at all analogous to Maxwell's propagation of wave disturbances in non-conductors, and it arises from his considering the water as contained in a tube like ordinary india-rubber tubes, instead of in a tube bored in an indefinitely large lump of india-rubber. If we consider this case it is evident that one of the conditions to be considered is the propagation of waves in this lump of india-rubber. In Sir Wm. Thomson's tube there would of course be a velocity of wave propagation in the india-rubber, but that is a very different matter from the propagation of disturbances away from the neighbourhood of the tube by which energy would be carried away from it. To do this Sir Wm. Thomson should have included the propagation of sound in the air or whatever he supposed surrounding the outside of his tube. Without including this, he was not including anything a bit analogous to Maxwell's electromagnetic theory of light. In Sir Wm. Thomson's tube the whole state of affairs at any time could be expressed in terms of variables that represented bodies near the tube, while in the other case it would be absolutely necessary to introduce variables representing every part of the india-rubber which I have supposed of indefinite extent. This is just the difference between Sir Wm. Thomson's and Maxwell's views. According to Maxwell's view there is a great deal more going on outside the conductor than inside it, and it is evident that the inertia of the water is a very bad analogue to electromagnetic induction, for this latter depends essentially upon the form of the circuit, and not only upon its section and length. Maxwell has shown that light may be a wave-propagation of what are on his theory analogous, though probably utterly unlike the distorsional waves propagated in the india-rubber, and has shown that a medium which would only transmit disturbances analogous to these would explain electric and magnetic phenomena. It is to be remembered that Maxwell's theory gets rid of all action at a distance, and that the only experimentum crucis between theories of action at a distance and of action through a medium is that in this latter case the energy may be propagated in time through the medium, while in the former it cannot.

I cannot conclude without protesting strongly against Sir Wm. Thomson's speaking of the ether as like a jelly. It is in some respects analogous to one, but we certainly know a great deal too little about it to say that it is like one. May be Maxwell's conceptions as to its structure are not very definite, but neither are any body's as to the actual structure of a jelly, and there is no real difficulty in supposing a medium whose condition is 